\title{
Determination of the Parameters of the Trajectory of Movement of a Standard Tool in Multi-Coordinate Machining of Spatially Modified Teeth of Spur Wheels with the Copying Technique
}

\author{
Roman Grubka ${ }^{1}$, Alexander Mikhaylov ${ }^{1}$, Irina Petryaeva ${ }^{1,}$, , Natalya Pichko $^{2}$, and Larisa \\ Polyakova $^{3}$ \\ ${ }^{1}$ Donetsk National Technical University, 283001, Artyoma str. 58, Donetsk, Ukraine \\ ${ }^{2}$ Usinsk branch of Ukhta State Technical University, 169710, Neftyanikov st. 33, Komi Republic, \\ Usinsk, Russia \\ ${ }^{3}$ Vorkuta branch of Ukhta State Technical University, 169900, Lenin st. 44, Komi Republic, Usinsk, \\ Russia
}

\begin{abstract}
The article presents a methodology for determining the parameters of the spatial trajectory of movement of a standard cutting tool processing ring gears with spatially modified teeth working by the copying technique. The implementation of the methodology makes it possible to determine the reference points coordinates of the trajectory of movement of the cutting tool in the process of multiple-axis processing of spatially modified teeth of spur wheels on CNC machines. The results obtained in the development of the methodology for determining the trajectory of the cutting tool movement can be further used in the process of performing the technological preparation for the production of spatially modified ring gears, including large-scale ones, in the conditions of single and smallscale production, as well as with choosing a technological equipment, the appointment of cutting modes, as well as in the process of writing control programs for $\mathrm{CNC}$ machines.
\end{abstract}

Spur wheels are made with an involute profile, and the teeth of one of the links are modified to increase the load capacity and durability of spur wheels $[1,2]$. Theoretically, the exact spatial modification of the teeth is obtained when two conditions of the second Olivier method are fulfilled, which is practically realizable in the operations of the ring gears finishingmachining. Ring gears with a spatial teeth modification close to the given spatial modification can be used for less significant gear-systems or can be cut with roughing machining the gears with an allowance for subsequent processing $[3,4]$.

In the process of cutting teeth, the involute profile is reproduced in the profiling plane perpendicular to the trajectory of the cutting tool. When cutting teeth with a straight generatrix,

\footnotetext{
*Corresponding author: irina_petryaeva@mail.ru
} 
the profiling plane is perpendicular to the axis of rotation of the workpiece and the involute is formed in the end section of the ring gear. When cutting helical gears, the profiling plane is located at an angle to the axis of rotation of the workpiece, and the position of the involute being cut changes accordingly. The teeth with a longitudinal modification are obtained, as a rule, by displacing the tool in the radial direction $[5,6]$, and since the profiling plane is perpendicular to

\section{DEFINITION OF SOURCE DATA}

2. PRELIMINARY DETERMINATION OF THE CT TRAJECTORY POINTS COORDINATES

3. PREDETERMINATION OF THE PARAMETERS OF THE PROFILING PLANE

\section{DETERMINATIONOF THE POINTS COORDINATES OF THE PROFILING PLANE INTERSECTION WITH THE TOOTH LATERAL SURFACE}

5. DEFINING TRANSITION PARAMETERS FROM $x_{i} y_{i} z_{i}$ к $x_{i} y_{0} z_{\theta}$

6. DETERMINATION OF THE POINTS COORDINATES ON THE TOOTH LATERAL SURFACE IN THE CT COORDINATE SYSTEM

7. CALCULATION OF THE STANDART TOOL CUTTING EDGE POINTS COORDINATES

8. DETERMINATION OF THE STANDART CT RADIAL CORRECTION VALUE

9. DETERMINATION OF THE STANDART CT TANGENTIAL CORRECTION VALUE

10. SPECIFYING THE POINTS COORDINATES OF THE CT MOVEMENT TRAJECTORY

11. APPROXIMATION OF THE POINTS COORDINATES OF THE CT MOVEMENT TRAJECTORY BY THE BEZIER SPLINE

12. DETERMINATION OF THE PROFILING PLANE PARAMETERS

13. REFINEMENT OF THE POINTS COORDINATES

OF THE PROFILING PLANE INTERSECTION WITH THE TOOTH LATERAL SURFACE

14. DEFINING TRANSITION PARAMETERS FROM $x_{l} y_{l} z_{1}$ K $x_{0} y_{0} z_{0}$

15. DETERMINATION OF THE UPDATED POINTS COORDINATES ON THE TOOTH LATERAL SURFACE IN THE CT COORDINATE SYSTEM

16. PROFILING NON-STANDARD CUTTING TOOLS

17. EVALUATION OF THE CUT GEOMETRY ACCURACY OF THE TOOTH LATERAL SURFACE FOR STANDART CT

Fig. 1. Method for the parameters determining of the cutting tool spatial trajectory. the trajectory of the relative movement of the cutting tool, the angle of inclination of the profiling plane will change accordingly. Thus, in the process of shaping the teeth, the involute profile is reproduced in the profiling plane, and the magnitude and direction of the displacement of the involute profile depends on the type of the cutting tool movement path [7]. In accordance with the rearranged classification of cutting tool movements [7] and shaping methods [8], in order to increase the accuracy of manufacturing ring gears with spatially modified teeth by the copying technique, the cutting tool must move along a spatial curvilinear trajectory, while there is a continuous change in position in space of the profiling plane.

When using the existing serial equipment and a standard tool working according to the copying technique, a ring gear is cut with a spatial modification of the teeth close to the theoretically calculated one. The degree of approximation of the geometric parameters of the lateral surface of the teeth to the theoretically calculated ones depends on the geometry of the cutting edge of the tool and the parameters of its movement's trajectory. The aim of the presented work is to develop a methodology for determining the parameters of the movement trajectory of a standard tool in multiple-axis machining of spatially modified teeth of spur wheels by copying technique.

For the technological methods of teeth shaping presented in [8], we will develop a method for determining the parameters of the tool path, when processing ring gears by copying technique with a complex spatial modification of the teeth $[1,9,10]$ with a standard cutting tool. The parameters of the trajectory of the relative movement of the cutting tool are determined depending on the geometry of the tool's cutting edge and the geometry of the side surface of the teeth of the ring gear being cut. In accordance with Figure 1 the 
sequence for determining the parameters of the movement trajectory of the cutting tool contains several sequentially performed stages.

As the initial data for determining the parameters of the trajectory of the tool working by the copying technique, there is the data obtained in approximating the geometry of the lateral surface of the tooth with spatial modification by the third-order Bézier splines [11].

At the first stage,the coordinates of the points of the spatial trajectory of movement of the cutting tool are preliminarily determined based on the maximum change in the thickness and height of the tooth in the section of the indexing cylinder, in the planes of symmetry and the end face of a ring gear:

$$
\left.\begin{array}{l}
x_{m p i}^{n p}=x_{m p}^{H}+\Delta x \cdot i / n \\
y_{m p i}^{n p}=y_{m p}^{H}-\Delta y \cdot i / n ; \\
z_{m p i}^{n p}=\frac{b_{w} i}{2 n} ; i=0,1, \ldots, n,
\end{array}\right\}
$$

where $x_{m p}^{H}$ and $y_{m p}^{H}$ are the coordinates of a point belonging to the symmetry plane of the ring gear;

$\Delta x$ and $\Delta y$ are respectively, the maximum deviations of the coordinates of the lateral surface points of the tooth in the section of the indexing cylinder in the tooth thickness and height;

$b_{w}$ is the width of the ring gear; $n$ is the number of points on the trajectory.

Having chosen the number of points (for example $n=10$ ) on the trajectory according to the dependence (1) the distribution of the spatial trajectory points' coordinates of the cutting tool movement is calculated. By connecting the points with segments, we obtain a spatial polyline, which pre-determines the geometry of the cutting tool spatial trajectory (Figure 2).

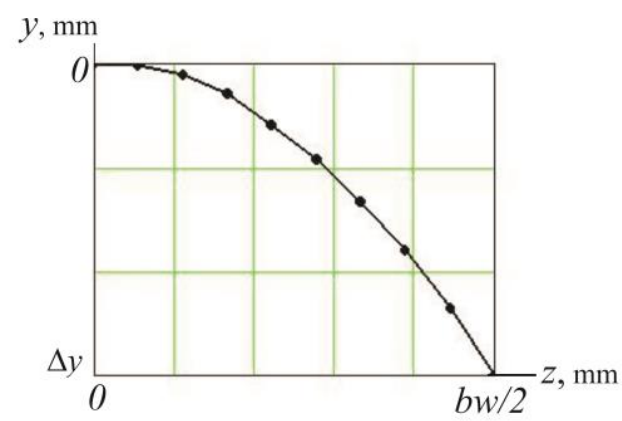

a)

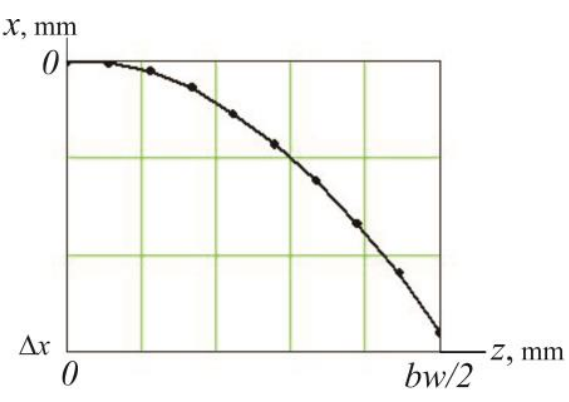

б)

Fig. 2. Preliminary calculating results of the tool trajectory points coordinates: a - projection onto the $\mathrm{yOz}$ plane; $\mathrm{b}$ - projection onto the $\mathrm{xOz}$ plane.

As shown in [8], the cutting tool profile is copied without distortion in the profiling plane perpendicular to the trajectory of its movement. The intersection points coordinates of the profiling plane with the spatially modified tooth lateral surface are determined by solving the system of equations [11]: 


$$
\left.\begin{array}{l}
x_{1}(\lambda)=x_{n_{-} k} \cdot(1-\lambda)^{3}+3 \cdot x_{1} \cdot \lambda \cdot(1-\lambda)^{2}+3 \cdot x_{2} \cdot \lambda^{2} \cdot(1-\lambda)+x_{n_{-} k} \cdot \lambda^{3}, \\
z_{1}(\lambda)=z_{n_{-} k} \cdot(1-\lambda)^{3}+3 \cdot z_{1} \cdot \lambda \cdot(1-\lambda)^{2}+3 \cdot z_{2} \cdot \lambda^{2} \cdot(1-\lambda)+z_{n_{-} k} \cdot \lambda^{3}, \\
A_{p i} \cdot x_{1}(\lambda)+B_{p i} \cdot y_{1 t e o r}+C_{p i} \cdot z_{1}(\lambda)+D_{p i}=0
\end{array}\right\}
$$

Where $x_{1}(\lambda)$ and $z_{1}(\lambda)$ are the points coordinates belonging to the spline;

$\lambda$ is the spline parameter;

$x_{n_{-} k}, x_{k_{-} k}, z_{n_{-} k}, \mathrm{z}$ respectively, the coordinates of the start and end points on the curve describing the gear tooth line;

$x_{1}, x_{2}, z_{1}, z_{2}$ are the spline control points coordinates;

$A_{p i}, B_{p i}, C_{p i}, D_{p i}$ arethe constant coefficients in the plane equation, determined by the dependencies for a trajectory straight line segment:

$$
\begin{gathered}
A_{p i}=x_{m p i+1}^{n p}-x_{m p i}^{n p} \\
B_{p i}=y_{m p i+1}^{n p}-y_{m p i}^{n p} \\
C_{p i}=z_{m p i+1}^{n p}-z_{m p i}^{n p}, \\
D_{p i}=0,5\left(\left(x_{m p i}^{n p}\right)^{2}+\left(y_{m p i}^{n p}\right)^{2}+\left(z_{m p i}^{n p}\right)^{2}-\left(\left(x_{m p i+1}^{n p}\right)^{2}+\left(y_{m p i+1}^{n p}\right)^{2}+\left(z_{m p i+1}^{n p}\right)^{2}\right)\right) .
\end{gathered}
$$

The parameters of the transition from the gear coordinate system $x_{1} y_{l} z_{l}$ to the cutting tool coordinate system are determined for each point of the trajectory according to the dependencies [12]:

$$
\begin{gathered}
\alpha_{x i}=\arctan \left(\frac{B_{p i}}{C_{p i}}\right) ; \\
\alpha_{y i}=\arctan \left(\frac{A_{p i}}{B_{p i} \sin \alpha_{x i}+C_{p i} \cos \alpha_{x i}}\right) ;
\end{gathered}
$$

The intersection points coordinates of the profiling plane with the spatially modified toothlateral surface, obtained as a result of solving the system of equations (2), are reduced to the cutting toolcoordinate system, taking into account the found angles $\alpha_{x}$ and $\alpha_{y}$ using the system of the following equations:

$$
\left.\begin{array}{l}
x_{0}=\cos \alpha_{y} x_{1}-\sin \alpha_{x} \sin \alpha_{y} y_{1}-\cos \alpha_{x} \sin \alpha_{y} z_{1}+z_{c p i} \sin \alpha_{y} \cos \alpha_{x}-x_{c p i} \cos \alpha_{y}+y_{c p i} \sin \alpha_{x} \sin \alpha_{y} \\
y_{0}=\cos \alpha_{x} y_{1}-\sin \alpha_{x} z_{1}+z_{c p i} \sin \alpha_{x}-y_{c p i} \cos \alpha_{x} \\
z_{0}=\sin \alpha_{y} x_{1}+\cos \alpha_{y} \sin \alpha_{x} y_{1}+\cos \alpha_{x} \cos \alpha_{y} z_{1}+-x_{c p i} \sin \alpha_{y}-y_{c p i} \cos \alpha_{y} \sin \alpha_{x}-z_{c p i} \cos \alpha_{x} \cos \alpha_{y} .
\end{array}\right\}
$$


The points coordinates on the standard tool cutting edge are determined by the dependencies [13]:

- for the left tooth profile:

$$
\left.\begin{array}{l}
x_{0 l}=r_{b 1}\left[\sin \left(\frac{\pi}{z_{1}}+\phi_{1}-\varphi_{c_{-} 1}\right)-\phi_{1} \cos \left(\frac{\pi}{z_{1}}+\phi_{1}-\varphi_{c_{-} 1}\right)\right], \\
y_{0 l}=r_{b 1}\left[\cos \left(\frac{\pi}{z_{1}}+\phi_{1}-\varphi_{c_{-} 1}\right)+\phi_{1} \sin \left(\frac{\pi}{z_{1}}+\phi_{1}-\varphi_{c_{-} 1}\right)\right] .
\end{array}\right\}
$$

- for the right tooth profile:

$$
\left.\begin{array}{l}
x_{0 p}=-r_{b 1}\left[\sin \left(\frac{\pi}{z_{1}}+\phi_{1}-\varphi_{c_{-} 1}\right)-\phi_{1} \cos \left(\frac{\pi}{z_{1}}+\phi_{1}-\varphi_{c_{-} 1}\right)\right], \\
y_{0 p}=r_{b 1}\left[\cos \left(\frac{\pi}{z_{1}}+\phi_{1}-\varphi_{c_{-} 1}\right)+\phi_{1} \sin \left(\frac{\pi}{z_{1}}+\phi_{1}-\varphi_{c_{-} 1}\right)\right] .
\end{array}\right\}
$$

where $r b l$ is the radius of the gear main circle;

$z_{1}$ is the gear teeth number;

$\varphi_{1}$ is the angle of the involute gear profile;

$\varphi_{c_{-} l} l$ is the offset angle of the gear involute beginning.

To refine the spatial trajectory parameters the radial and tangential correction values of the cutting tool position are entered.

The radial correction value is determined by solving the system of equations (8), taking into account that the gear tooth height coordinate changes by the correction value. And in the left part of the equations system (8), the points coordinates belonging to the gear tooth lateral surface are substituted, reduced to the cutting tool coordinate system according to the dependencies (6):

$$
\left.\begin{array}{l}
x_{0 i}=-r_{b 1}\left[\sin \left(\frac{\pi}{z_{1}}+\phi_{1}-\varphi_{c_{-} 1}\right)-\phi_{1} \cos \left(\frac{\pi}{z_{1}}+\phi_{1}-\varphi_{c_{-} 1}\right)\right], \\
y_{0 i}=r_{b 1}\left[\cos \left(\frac{\pi}{z_{1}}+\phi_{1}-\varphi_{c_{-} 1}\right)+\phi_{1} \sin \left(\frac{\pi}{z_{1}}+\phi_{1}-\varphi_{c_{-} 1}\right)\right]+k_{r i} \cdot
\end{array}\right\}
$$

The tangential correction value is determined by the dependence:

$$
k_{t j}=0,5\left(\delta x_{00}+\delta x_{0 n}\right) \text {. }
$$

where $\delta x_{00}$ and $\delta x_{0 n}$ - is the deviation of the tooth thickness for the upper and lower points of the profile:

$$
\delta x_{0 i}=x_{0 i}^{m e o p}-x_{0 i}
$$


where $x_{0 i}^{\text {meop }}$ and $x_{0 i}$ - coordinates along the thickness of the tooth, theoretical and corresponding to the theoretical values of the thickness at the boundary points of the tooth profile:

$$
x_{0 i}=-r_{b 1}\left[\sin \left(\frac{\pi}{z_{1}}+\phi_{1 i}-\varphi_{c_{-} 1}\right)-\phi_{1 i} \cos \left(\frac{\pi}{z_{1}}+\phi_{1 i}-\varphi_{c_{-} 1}\right)\right],
$$

where $\phi_{11}$ and $\phi_{12}$ - the unfolding angles of the involute for the boundary curve points of intersection of the lateral surface of the spatially modified tooth with the profiling plane, determined taking into account the previously found values of the radial correction, by solving the system of equations:

$$
\left.\begin{array}{l}
y_{00}=r_{b 1}\left[\cos \left(\frac{\pi}{z_{1}}+\phi_{11}-\varphi_{c_{-} 1}\right)+\phi_{11} \sin \left(\frac{\pi}{z_{1}}+\phi_{11}-\varphi_{c_{-} 1}\right)\right]+k_{r i} \\
y_{0 n}=r_{b 1}\left[\cos \left(\frac{\pi}{z_{1}}+\phi_{12}-\varphi_{c_{-} 1}\right)+\phi_{12} \sin \left(\frac{\pi}{z_{1}}+\phi_{12}-\varphi_{c_{-} 1}\right)\right]+k_{r i} \cdot
\end{array}\right\}
$$

The points coordinates of the actually cut involute are determined in accordance with the system of equations:

$$
\left.\begin{array}{l}
x_{0 i, j}^{\text {uap }}=-r_{b 1}\left[\sin \left(\frac{\pi}{z_{1}}+\phi_{1 i}-\varphi_{c_{-} 1}\right)-\phi_{1 i} \cos \left(\frac{\pi}{z_{1}}+\phi_{1 i}-\varphi_{c_{-} 1}\right)\right]-k_{t j}, \\
y_{0 i, j}^{\text {uap }}=r_{b 1}\left[\cos \left(\frac{\pi}{z_{1}}+\phi_{1 i}-\varphi_{c_{-} 1}\right)+\phi_{1 i} \sin \left(\frac{\pi}{z_{1}}+\phi_{1 i}-\varphi_{c_{-} 1}\right)\right]+k_{r j} .
\end{array}\right\}
$$

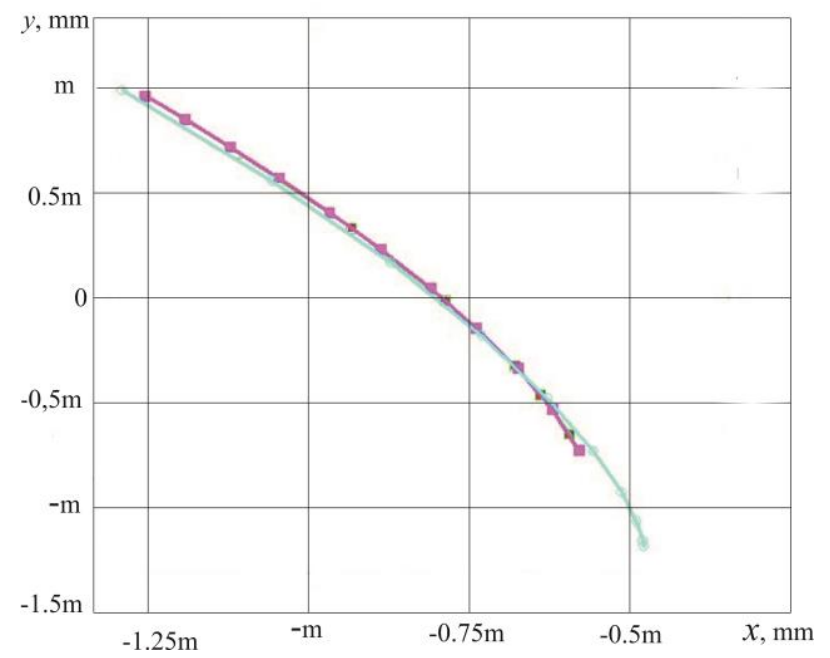

Fig. 3. Tooth profiles and cut involute in the profiling plane.

Based on the calculations results using dependencies (14), we construct theoretical curves graphs of the involute curve being cut and the spatially modified tooth lateral surface section by a profiling plane, reduced to the cutting tool coordinate system and (Figure 3.). 
At the second stage, the specified points coordinates of the cutting tool spatial displacement trajectory are determined, taking into account the previously found values of the radial and tangential cutting tool position corrections:

$$
\left.\begin{array}{l}
x_{i}^{m p}=x_{m p i}^{n p}-k_{t i} ; \\
y_{i}^{m p}=y_{m p i}^{n p}+k_{r i} ; \\
z_{i}^{m p}=z_{m p i}^{n p} .
\end{array}\right\}
$$

The points coordinates approximation of the cutting tool spatial trajectory movement is carried out by a third-order Bézier spline:

$$
\left.\begin{array}{l}
x_{i}^{m p}=\left(1-\lambda_{i}^{m p}\right)^{3} x_{0}^{m p}+3 \cdot\left(1-\lambda_{i}^{m p}\right)^{2} \lambda_{i}^{m p} x 1_{m p}+3 \cdot\left(1-\lambda_{i}^{m p}\right) \lambda_{i}^{m p 2} x 2_{m p}+\lambda_{i}^{m p 3} x_{n}^{m p} ; \\
y_{i}^{m p}=\left(1-\lambda_{i}^{m p}\right)^{3} y_{0}^{m p}+3 \cdot\left(1-\lambda_{i}^{m p}\right)^{2} \lambda_{i}^{m p} y 1_{m p}+3 \cdot\left(1-\lambda_{i}^{m p}\right) \lambda_{i}^{m p 2} y 2_{m p}+\lambda_{i}^{m p 3} y_{n}^{m p} ; \\
z_{i}^{m p}=\left(1-\lambda_{i}^{m p}\right)^{3} z_{0}^{m p}+3 \cdot\left(1-\lambda_{i}^{m p}\right)^{2} \lambda_{i}^{m p} z 1_{m p}+3 \cdot\left(1-\lambda_{i}^{m p}\right) \lambda_{i}^{m p 2} z 2_{m p}+\lambda_{i}^{m p 3} z_{n}^{m p}
\end{array}\right\}
$$

where $x_{i}^{m p}, y_{i}^{m p}, z_{i}^{m p}$ are the $i$ point coordinates of the cutting tool trajectorymovement; $x_{0}^{m p}, y_{0}^{m p}, z_{0}^{m p}$ and $x_{n}^{m p}, y_{n}^{m p}, z_{n}^{m p}$ are the points coordinates of the cutting tool trajectory movement beginning and end;

$x 1_{m p}, y 1_{m p}, z 1_{m p}$ and $x 2_{m p}, y 2_{m p}, z 2_{m p}$ are coordinates of the spline control points;

$\lambda_{i}^{m p}$ is the spline i-parameter.

In further considering the found cutting tool trajectory movement parameters refining coordinates calculations of the profiling plane intersection with the lateral surface of the tooth with spatial modification are performed. Coordinate calculations are performed using the system of equations (2), taking into account the plane parameters perpendicular to the spatial curve of the cutting tool trajectory movement found from dependencies (3). The transition parameters $\alpha_{x}$ and $\alpha_{y}$ from the $x_{1} y_{l} z_{1}$ coordinate system to the tool coordinate system are calculated using dependencies (4) and (5). After that, the refined points coordinates values of the profiling plane intersection with the lateral surface of the spatially modified tooth are brought to the cutting tool coordinate system according to the dependencies (6).

The quality of the calculations performed to determine the parameters of the cutting tool spatial movement trajectory is checked by assessing the accuracy of obtaining the coordinates of the spatially modified gear tooth lateral surface. For this, the theoretical values of the points coordinates on the spatially modified teeth lateral surface are compared with the coordinates that will be obtained during machining if the cutting tool moves relative to the workpiece along the calculated trajectory without taking into account the elements deformations of the "machine - fixture - tool - detail" system.

To assess the accuracy of the cut teeth side surface geometry, we substitute the theoretical value of the coordinate along the tooth height, reduced to the cutting tool coordinate system in one of the system equations (14) $y_{0 i, j}^{\text {meop }}$ : 


$$
y_{0 i, j}^{\text {meop }}=r_{b 1}\left[\cos \left(\frac{\pi}{z_{1}}+\phi_{1 i}-\varphi_{c_{-} 1}\right)+\phi_{1 i} \sin \left(\frac{\pi}{z_{1}}+\phi_{1 i}-\varphi_{c_{-} 1}\right)\right]+k_{r j} .
$$

From the obtained in this way equation, we determine the value of the involute unfolding angle $\phi_{1 i}$, corresponding to the current value of the coordinate along the height of the tooth $y_{0 i, j}^{\text {meop }}$. After substituting the value of the involute unfolding angle $\phi_{1 i}$ into the first equation of system (14), we calculate the value of the point coordinate belonging to the being cut involute during processing:

$$
x_{0 i, j}^{\text {нap }}=-r_{b 1}\left[\sin \left(\frac{\pi}{z_{1}}+\phi_{1 i}-\varphi_{c_{-} 1}\right)-\phi_{1 i} \cos \left(\frac{\pi}{z_{1}}+\phi_{1 i}-\varphi_{c_{-} 1}\right)\right]-k_{t j}
$$

We carry out a quantitative assessment of the ring gear shaping accuracy, taking into account the values of the absolute and relative deviations of the teeth actually cut side surface points coordinates from the theoretically calculated ones.

Thus, a method has been developed for determining the dependencies that describe the cutting tool spatial trajectory operating by the copying technique while processing ring gears with spatially modified teeth.The implementation of the technique allows to determine the control points coordinates of the cutting tool spatial movement trajectory in the process of multiple-axis processing on $\mathrm{CNC}$ machines of the spur wheels spatially modified teeth.The method assumes the use of a standard cutting tool during processing.The results obtained in the development of a determining method for the cutting tool movement trajectory can be further used in calculations related to the designation of processing parameters.

The proposed approaches can be used in the ring gearsprocessing, including large-scale ones, in the conditions of single and small-scale production while choosing technological equipment and cutting tools, as well as in the process of writing control programs for $\mathrm{CNC}$ machines.

The use of the proposed method for determining the parameters of the cutting tool spatial trajectory allows to increase the accuracy of the ring gears multiple-axis milling due to the analytical description of the correlation between the geometry of the spatially modified teeth lateral surface, the geometry of the tool cutting edge and the cutting tool movement trajectory relative to the workpiece.

\section{References}

1. Grubka, R. M. Synthesis of spatial geometries of teeth of cylindrical wheels operating in the presence of errors and deformations of gear elements [Text] / R. M. Grubka, I. A. Petryaeva, A. N. Mikhailov, A. A. Bocharov // Progressive technologies and systems of mechanical engineering: International collection. scientific papers. Donetsk: DonNTU, 2018. - Issue. 4 (63). - P. 17 - 27.

2. Grubka, R.M. Constructive ways of improving the performance of cylindrical gears [Text] / RM. Grubka // Progressive technologies and systems of mechanical engineering: International collection. scientific papers. - Donetsk: DNTU, 2019. Issue. 1 (64). - P. 17 - 22.

3. Grubka, R. M. Methods for determining the parameters for setting up a gear hobbing machine when milling the teeth of the bushings of gear couplings with spatial 
geometry [Text] / R. M. Grubka, A. N. Mikhailov, S. I. Luchko // Progressive technologies and systems of mechanical engineering: International science - Donetsk: DNTU, 2011. - Issue. 41 .- P. 112-118.

4. Grubka, R. M. Milling of the teeth of the sleeve bushings with a geometry close to the group spatial method of running-in on the existing serial equipment [Text] / R. M. Grubka, A. N. Mikhailov, L. N. Fenik, S. I. Luchko / / Progressive technologies and systems of mechanical engineering: International collection. scientific papers. Donetsk: Donets.nats. tech. un-t, 2011. - Issue. 42 .- P. 98-103.

5. Patent 944824 USSR, IPC V 23 F $9 / 00$ Method of processing gear wheels / V.A. Danilov; applicant and patentee Novopolotsk Polytechnic Institute. - No. 3005693 / 25 08; declared 11/19/80; publ. 07/23/82, Bul. No. 27. - 2 p. : ill.

6. Patent 685451 USSR, IPC V $23 \mathrm{~F} 1 / 00$ Method of processing gear wheels / N.E. Ternyuk, V.B. Varshavsky, A.A. Chlus. - No. 2418078 / 25-08; declared 11/04/76; publ. 09/15/1979. Bul. No. 34. - 2 p. : ill.

7. Grubka, R. M. Classification of movements in multi-axis gear milling of spatially modified teeth of cylindrical gears by copying [Text] / R. M. Grubka, A. N. Mikhailov, I. A. Petryaeva // Progressive technologies and systems of mechanical engineering: International Sat. scientific papers. - Donetsk: Donets. nat. tech. un-t, 2019. - Issue. 3 (66). - P. 16 - 21.

8. Gear hobbing by copying cylindrical wheels with spatially modified teeth / R.M. Grubka, A.N. Mikhailov, A.V. Stepochkina, I.A. Gurichev // Perspective directions of development of finishing and hardening treatment and vibration wave technologies [Electronic resource]: collection of articles. tr. scientific. seminar / ed. V.A. Lebedev; Don state tech. un-t. - Electron. text data. - Rostov-on-Don: DSTU, 2021 .- P. 242 - 247.

9. Patent 2713537 Rus. Federation, MKP7 F16H 1/04 (2006.01). Toothed connection with external gear teeth / R.M. Grubka, N.S. Pichko, A.N. Mikhailov, I.A. Petryaeva; applicant and patentee Federal State Budgetary Educational Institution of Higher Education "Ukhta State Technical University" (FGBOU VO USTU) (RU) - No. 2019104782, app. 02/20/2019; publ. 02/05/2020 Bul. No. 4 - 11 p. : ill.

10. Patent 2713691 Rus. Federation, MKP7 F16H 1/10 (2006.01) F16D 3/18 (2006.01). Toothed connection with internal gear teeth / R.M. Grubka, N.S. Pichko, A.N. Mikhailov, I.A. Petryaeva; applicant and patentee Federal State Budgetary Educational Institution of Higher Education "Ukhta State Technical University" (FGBOU VO USTU) (RU) - No. 2019104785, app. 04/10/2019; publ. 02/06/2020 Bul. No. 4 - 13 p. : ill.

11. Some features of the design and technological preparation of the production of spur gears with spatially modified teeth / R.M. Grubka, N.S. Pichko, I.A. Petryayeva, A.N. Mikhailov // Materials Today: Proceedings. - 2019. - Volume 19. Part 5: International Conference on Modern Trends in Manufacturing Technologies and Equipment 2019. P. 2483-2487.

12. Grubka, R. M. Determination of the transition parameters between coordinate systems of the cutting tool and the workpiece in multi-coordinate machining of cylindrical wheels [Text] / R. M. Grubka // Progressive technologies and systems of mechanical engineering: International collection. scientific papers. - Donetsk: Donets. nat. tech. un-t, 2021. - Issue. 2 (73). - P. 34 - 41.

13. Parts of machines [Text]: studies. for universities / L. A. Andrienko [and others]. - 3rd ed., Rev. and add. - M .: Publishing house of MSTU N.E. Bauman named, 2007 .- 520 p. 